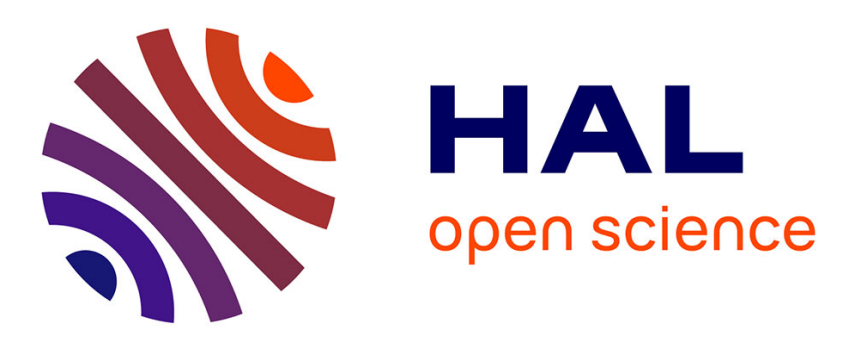

\title{
An application of the ince algebraization to the stability of non-linear normal vibration modes
}

\author{
Yuri Mikhlin, A. Zhupiev
}

\section{To cite this version:}

Yuri Mikhlin, A. Zhupiev. An application of the ince algebraization to the stability of non-linear normal vibration modes. International Journal of Non-Linear Mechanics, 1997, 32 (2), pp.393-409. 10.1016/S0020-7462(96)00047-9 . hal-01348301

\section{HAL Id: hal-01348301 \\ https://hal.science/hal-01348301}

Submitted on 22 Jul 2016

HAL is a multi-disciplinary open access archive for the deposit and dissemination of scientific research documents, whether they are published or not. The documents may come from teaching and research institutions in France or abroad, or from public or private research centers.
L'archive ouverte pluridisciplinaire HAL, est destinée au dépôt et à la diffusion de documents scientifiques de niveau recherche, publiés ou non, émanant des établissements d'enseignement et de recherche français ou étrangers, des laboratoires publics ou privés.

\section{(c)(1)}

Distributed under a Creative Commons Attribution| 4.0 International License 


\title{
AN APPLICATION OF THE INCE ALGEBRAIZATION TO THE STABILITY OF NON-LINEAR NORMAL VIBRATION MODES
}

\author{
Yu. V. Mikhlin* and A. L. Zhupiev† \\ *Department of Applied Mathematics, Kharkov Polytechnic University, 310002, Kharkov, \\ Ukraine; \\ $\dagger$ Department of Applied Mechanics, Mining Academy of Dnepropetrovsk, \\ 320600, Dnepropetrovsk, Ukraine
}

\begin{abstract}
A normal vibration mode stability in conservative non-linear systems is investigated. The algebraization by Ince (transition from linear equations with periodic coefficients to equations with singular points) is used. The normal mode stability in homogeneous systems, whose potential is an even homogeneous function of the variables and systems close to the homogeneous one, is investigated. Eigenvalues and eigenfunctions a re obtained. Conditions when a number of instability zones in a non-linear system parameters space are finite (finite zoning or finite-gap conditions) are also obtained.
\end{abstract}

Keywords: normal vibration mode, stability, bifurcation, Ince algebraization

\section{INTRODUCTION}

Normal (principal) vibration modes can be used to construct a general solution in the linear theory. In non-linear systems the "normal vibration modes" (or "normal vibrations") are defined as such free motions where all coordinates of the system vary equiperiodically, reaching the extreme equipotential surface simultaneously [1-4]. All position coordinates can be well defined from any one of them by

$$
x_{i}=p_{i}\left(x_{1}\right) \quad(i=2,3, \ldots)
$$

$p_{i}(x)$ being analytical functions.

Rosenberg [1,2] gets credit for being the first to introduce broad classes of essentially non-linear (not quasilinear) conservative systems allowing normal vibrations with rectilinear trajectories in a configurational space (similar normal modes). In his and other works, normal mode stability was computed by linearized, Mathieu-type analysis [1,2,5-7], but it cannot guarantee good precision.

The stability of the normal vibration modes is studied by deriving an approximation for the Poincare map by Month and Rand (via Birkhoff-Gustavson canonical transformation) [8] and Pak [9]. This method is presented as an alternative to the usual linearized stability on Floquet-Liapunov theory. Some papers $[9,10]$ are based on Synge's stability in the kinematico-statical sense. Some examples were considered. In Pecelli and Thomas's works $[11,12]$ the Poincare map, Kolmogorov-Arnold-Moser theorems and Lamé equation theory were used. The similar normal modes stability was investigated while the linearizing variational equations represent the Lamé equation; criterions of a stability in the non-linear sense were obtained. The effect of a normal mode bifurcation is investigated in various papers, for example, $[7,10]$. This effect and resonance neighborhood are investigated by numerical and analytical techniques in [13].

In this work, the algebraization by Ince [14] and transition from linear equations with periodic coefficients to equations with singular points (Section 2) will be used. The normal vibration modes stability of conservative homogeneous systems whose potential is an even homogeneous function of the variables and systems close to the homogeneous one will be investigated (Section 3). Eigenvalues and eigenfunctions will be obtained. Conditions when a number of instability zones in a system parameters space are finite (finite zoning or 
finite-gap conditions) will be also obtained (Section 4). Finally, in Section 5, the results relating to non-linear stability analysis $[11]$ will be reduced.

\section{TRANSITION FROM LINEAR EQUATIONS WITH PERIODIC COEFFICIENTS TO EQUATIONS WITH SINGULAR POINTS}

Let us now describe what is called the algebraization by Ince, that is the transfer from equations with periodic coefficients to equations with singular points, following [14], together with the relevant change in the stability problem. There are many methods of algebraization but only one of them is shown in this item. In the analytical treatment of normal vibrations, the algebraization is performed by choosing a new independent variable associated with the undisturbed motion. The variable $t$ may be replaced by $x$, a variable that defines motion along a rectilinear trajectory, or by the velocity $\dot{x}$, or by the kinetic energy for this solution etc.

We consider now the Hill equation

$$
\ddot{y}+\left(\sigma_{0}+2 \sigma_{1} \cos 2 t+\sigma_{2} \cos 4 t+\cdots\right) y=0
$$

Let us introduce a new independent variable $z=\cos t$. We obtain

$$
\left(1-z^{2}\right) \frac{\mathrm{d}^{2} y}{\mathrm{~d} z^{2}}-z \frac{\mathrm{d} y}{\mathrm{~d} z}+\left(\sum_{n=0}^{\infty} b_{n} z^{2 n}\right) y=0 .
$$

This equation has regular singularities at $z= \pm 1$ (the indices for singular points are 0 and $1 / 2$ ), and an irregular singular point at infinity.

Two fundamental solutions may be separated for the vicinity of the singular point $z=1$ $[14]:$

$$
y_{1}(1-z)=f_{1}(1-z) ; \quad y_{2}(1-z)=\sqrt{1-z} f_{2}(1-z),
$$

where $f_{1}$ and $f_{2}$ are analytical functions that converge within a circle $|1-z| \leqslant 2$.

Since the equation will not change if $z$ is replaced by $-z$, the following solutions will be associated with the singular point $z=-1$ :

$$
y_{1}(1+z)=f_{1}(1+z) ; \quad y_{2}(1+z)=\sqrt{1+z} f_{2}(1+z),
$$

Here the analytical functions $f_{1}$ and $f_{2}$ converge within a circle $|1+z| \leqslant 2$.

Within a common region of the two circles of convergence

$$
\begin{aligned}
& y_{1}(1-z)=\alpha y_{1}(1+z)+\beta y_{2}(1+z) \\
& y_{2}(1-z)=\gamma y_{1}(1+z)+\delta y_{2}(1+z),
\end{aligned}
$$

$\alpha, \beta, \gamma, \delta$ are constant.

Replacing $z$ by $-z$, one obtains

$$
\begin{aligned}
& y_{1}(1+z)=\alpha y_{1}(1-z)+\beta y_{2}(1-z)=\left(\alpha^{2}+\beta \gamma\right) y_{1}(1+z)+\beta(\alpha+\delta) y_{2}(1+z), \\
& y_{2}(1+z)=\gamma y_{1}(1-z)+\delta y_{2}(1-z)=\gamma(\alpha+\delta) y_{1}(1+z)+\left(\beta \gamma+\delta^{2}\right) y_{2}(1+z)
\end{aligned}
$$

It follows that $\alpha^{2}+\beta \gamma=\beta \gamma+\delta^{2}=1$ and $\beta(\alpha+\delta)=\gamma(\alpha+\delta)=0$, with only two cases possible: (1) $\alpha=\delta= \pm 1, \beta=\gamma=0$; and (2) $\alpha=-\delta, \beta \gamma=1-\alpha^{2}$. Let us consider the case of $\alpha=\delta=1, \beta=\gamma=0$. Here

$$
\begin{aligned}
& y_{1}(1-z)=y_{1}(1+z), \\
& y_{2}(1-z)=y_{2}(1+z) .
\end{aligned}
$$

The origin of coordinates, $z=0$, is, however, a regular point of the equation, therefore no two independent even solutions can exist that are meaningful in the vicinity of the point $z=0$. It follows that condition (1) must be rejected. The condition $\alpha=\delta=-1, \beta=\gamma=0$ is not suitable, since it is associated with two distinct even solutions that are meaningful in the origin of coordinates. Hence, only condition (2) remains. We shall restrict ourselves to the cases which will bring us to the $\pi$ - or $2 \pi$-periodic "boundary" solutions that separate the stability regions from the instability regions of the single equation with periodic coefficients $[15,16]$. 
(a) Let us assume that $\alpha=-\delta= \pm 1, \beta=0 ; y_{1}(1-z)= \pm y_{1}(1+z)$.

The solution is even at $\alpha=1$ and odd at $\alpha=-1$; it is regular in any finite part of the plane. Since $z=\cos t$, at $\alpha=1$ the solution may be presented as an even power expansion in cosines that are multiples of $t$, and $\alpha=-1$, in the form of an odd power expansion in cosines that are multiples of $t$.

(b) Let $\alpha=-\delta= \pm 1, \gamma=0, y_{2}(1-z)= \pm y_{2}(1+z)$ at $|1 \pm z|<2$.

The solution is the product of $\sqrt{1-z^{2}}$ by a regular function that changes its sign upon each revolution about the singular point $z=1$ or $z=-1$. The regular function is even if $\delta=1$ and odd if $\delta=-1$. Substituting $z=\cos t$, one obtains an even power expansion in sines that are multiples of $t(\delta=1)$, and an odd power expansion in sines that are multiples of $t(\delta=-1)$.

Thus, for the Hill equation in algebraic form, "boundary" solutions of the Hill equation are obtained that separate the region in which all solutions are bounded from the one in which all solutions are unbounded.

Let us consider two examples which are special cases of the Hill equation.

Mathieu equation: $\ddot{y}+(\delta+2 \varepsilon \cos 2 t) y=0$.

Substituting $z=(\sin t)^{2}$, one obtains an algebraic form of

$$
4(1-z) \frac{\mathrm{d}^{2} y}{\mathrm{~d} z^{2}}+2(1-2 z) \frac{\mathrm{d} y}{\mathrm{~d} z}+(\delta+2 \varepsilon-4 \varepsilon z) y=0 .
$$

It has two regular singular points: $z=0$ and $z=1$ whose indices are 0 and $1 / 2$, and an essential singular point $z=\propto$. In view of the above, the solutions separating the stability region from the instability region should be sought in the following form:

$$
\begin{gathered}
y^{(1)}=\sum_{k=1,3 \ldots}^{\infty} a_{k}^{(1)} z^{k}, \\
y^{(2)}=\sum_{k=0,2,4, \ldots} a_{k}^{(2)} z^{k}, \\
y^{(3)}=\sqrt{z(1-z)} \sum_{k=1,3, \ldots}^{\infty} a_{k}^{(1)} z^{k}, \\
y^{(4)}=\sqrt{z(1-z)} \sum_{k=0,2,4, \ldots} a_{k}^{(2)} z^{k},
\end{gathered}
$$

where each series converges in a domain containing both regular singular points. Using analytical or numerical methods, one can eventually obtain the Ince-Strett diagram.

Lamé equation: $\ddot{y}+\left\{h-n(n+1)[k \operatorname{sn}(t, k)]^{2}\right\} y=0$.

Here, a transfer to algebraic forms may be effected by a number of methods [17]. For example, substituting $z=[s n(t, k)]^{2}$, one obtains the following equation with three finite regular singular points:

$$
\frac{\mathrm{d}^{2} y}{\mathrm{~d} z^{2}}+\frac{1}{2}\left(\frac{1}{z}+\frac{1}{z-1}+\frac{1}{z-k^{-2}}\right) \frac{\mathrm{d} y}{\mathrm{~d} z}+\frac{h k^{-2}-n(n+1) z}{z(z-1)\left(z-k^{-2}\right)} y=0
$$

Algebraic forms of the Lamé equation are used in a number of problems considered in this article.

3. THE SIMILAR NORMAL MODES STABILITY BY THE FIRST APPROXIMATION OF HOMOGENEOUS SYSTEMS AND SYSTEMS CLOSE TO THE HOMOGENEOUS ONE

\subsection{Splitting of variational equations}

Let us consider variational equations for rectilinear vibration modes $x_{i}=k_{i} x_{1}$ $(i=2,3, \ldots, n)$ in a conservative system of the form

$$
m_{i} \ddot{x}_{i}+\Pi_{x_{i}}=0 \quad\left(\dot{x}_{i}=\frac{\mathrm{d} x_{i}}{\mathrm{~d} t}, \Pi_{z}=\frac{\partial \Pi}{\partial z}, i=1,2, \ldots, n\right),
$$

whose potential energy $\Pi\left(x_{1}, x_{2}, \ldots, x_{n}\right)$ is analytical. 
Let us rotate the coordinate axes so that the axis $x_{1}$ is aligned with the rectilinear trajectory, with the remaining axes orthogonal to the trajectory. In the new coordinates, the trajectory is defined as $x_{j}=0(j=2,3, \ldots, n), x_{1} \equiv x(t)$.

It was shown in [6] that upon such rotation of the axes in a system with two degrees of freedom the variational equations always "split". Denoting the variation along the rectilinear trajectory by $u$, and the variation in the orthogonal direction by $v$, we obtain:

$$
\begin{gathered}
\dot{u}+\frac{\mathrm{d}}{\mathrm{d} x} \Pi_{x}(x, 0) u=0, \\
\ddot{v}+\Pi_{x_{2} x_{2}}(x, 0) v=0 .
\end{gathered}
$$

Let us turn to a system with $n$ degrees of freedom. Introducing a vector of variations $\mathbf{U}=\left(u_{1}, u_{2}, \ldots, u_{n}\right)^{\mathrm{T}}$ : we obtain a set of variational equations in the matrix form

$$
\ddot{\mathbf{U}}+(\mathbf{U} \boldsymbol{\nabla}) \boldsymbol{\nabla} \boldsymbol{\Pi}=0
$$

or

$$
\ddot{\mathbf{U}}+\mathbf{A} \mathbf{U}=0 .
$$

Let us expand the matrix $\mathbf{A}$ is a series according to $x$ :

$$
\ddot{\mathbf{U}}+\left(\mathbf{A}_{0}+\mathbf{A}_{1} x+\mathbf{A}_{2} x^{2}+\cdots\right) \mathbf{U}=0,
$$

where $\mathbf{A}_{i}$ are symmetric matrices with constant coefficients.

For a homogeneous system whose potential is a homogeneous function of variables to the power $r+1$, equations (2) is "split" by the use of a non-degenerate transformation with constant coefficients. Indeed, the variational equations then have the form

$$
\ddot{\mathbf{U}}+\mathbf{A}_{\boldsymbol{r}} \mathbf{U}=0,
$$

and the symmetric matrix $\mathbf{A}_{r}$ can be diagonalized using a non-degenerate orthogonal transformation [18].

Let us separate out another case:

$$
\ddot{\mathbf{U}}+\left[\mathbf{A}_{n} x^{n}+\mathbf{A}_{m} x^{m}\right] \mathbf{U}=0 .
$$

The two symmetric matrices $\mathbf{A}_{n}$ and $\mathbf{A}_{m}$ are always diagonalizable through one nondegenerate transformation [18], provided one of them is positively defined; moreover, if the matrices are commuting, the transformation may be made orthogonal. In the literature [19] one can find proof of the theorem on the diagonalization in the general system (2) under the conditions that all matrices $\mathbf{A}_{i}$ commute between them and also have non-multiple proper values.

Let the set of variational equations decompose into $n$ independent equations. One of these will govern the variations along the rectilinear trajectory, and the remaining equations will refer to the orthogonal directions. It is with the variations in the orthogonal directions that the orbital stability of normal vibrations is associated.

Let us introduce a new independent variable $x$. Every variational equation then will have the following form where the primes refer to the derivation with respect to $x$ :

$$
2 S y^{\prime \prime}+S^{\prime} y^{\prime}+G(x) y=0, \quad S=(a-x)(b-x) R .
$$

Here $R$ is an analytical function that has no real roots whose moduli are smaller than $a$ or $b$. As shown in the previous section, the problem of defining $\tau$ - and $2 \tau$-periodic solutions of the variational equations ( $\tau$ is the period of the coefficients in the equations) that separate the regions of stability and instability is reduced to solving Sturm-Liouville problems for functions that are regular at $x=a$ and $x=b$ or have a singularity of the form $\sqrt{a-x}$ or $\sqrt{b-x}$ at these points.

If the potential energy $\Pi$ is an even analytical function, the coefficients of the variational equations have a period which is twice as short as that of the normal vibrations. The kinetic energy is proportional to $h-\Pi$ and is an even function of $x$ too. However, $S$ in (3) is proportional to the kinetic energy, therefore here $b=-a$. Without loss of generality, one thus can select a scale such that $S=\left(1-x^{2}\right) R$. The problem of defining the boundaries of 
the stability and instability regions by the first approximation is now decomposed into four Sturm-Liouville problems for even and odd functions that are regular at $x= \pm 1$ or have a singularity of the form $\sqrt{1-x^{2}}$ at these points.

\subsection{Homogeneous systems}

Let us consider variational equations for homogeneous systems with the potential energy

$$
\Pi=\sum_{\substack{\alpha_{1}+x_{2}+\ldots, x_{n}=r+1 \\ \alpha_{i}=0,1,2, \ldots, r+1}} \frac{\alpha_{1} \alpha_{2} \cdots \alpha_{n}}{r+1} x_{1}^{\chi_{1}} x_{2}^{\chi_{2}} \cdots x_{n}^{\chi_{n}}
$$

Upon the diagonalization of the matrix $\mathbf{A}$ each of the equations can be written in the form

$$
\ddot{v}+q x^{r-1} v=0 \quad(q=\text { const. })
$$

Here

$$
\begin{gathered}
\ddot{x}=-\Omega^{2} x^{r}, \quad \dot{x}^{2}=2\left(h-\Omega^{2} \frac{x^{r+1}}{r+1}\right), \\
\Omega^{2}=\pi\left(1, k_{2}, \ldots, k_{n}\right) .
\end{gathered}
$$

Let us replace $t$ by a new independent variable $x$. We obtain, instead of (5),

$$
v^{\prime \prime} \dot{x}^{2}+v^{\prime} \ddot{x}+q x^{r-1} v=0
$$

or

$$
2 v^{\prime \prime}\left(h-\Omega^{2} \frac{x^{r+1}}{r+1}\right)-v^{\prime} \Omega^{2} x^{r}+q x^{r-1} v=0
$$

Let us introduce another variable $z=\Omega^{2} x^{r+1} /(r+1)$. We then have

$$
z(1-z) v_{z z}+\left[\frac{r}{r+1}-\frac{3 r+1}{2(r+1)} z\right] v_{z}+\dot{\lambda} v=0
$$

where

$$
\lambda=\frac{q}{2(r+1) \Omega^{2}} .
$$

Relationship (6) is a hypergeometric equation with regular singular points $z=0$ (the indices equal 0 and $1 /(r+1))$ and $z=1$ (the indices equal 0 and $1 / 2$ ). The problem of stability and branching, as suggested above, is now much simpler. It essentially reduces to finding the values of the parameter $i$ such that, on having traversed a closed contour containing singular points 0 and 1 , a solution is multiplied by +1 or by -1 (Section 2). Such solutions are named degenerate solutions of the hypergeometric equation and are given in [20]. Their form is

$$
v=z^{\mu_{1}}(1-z)^{\mu_{2}} q_{n}(z),
$$

where $q_{n}(z)$ is a polynome, $\mu_{1}$ can take values of 0 and $1 /(r+1), \mu_{2}$ can take values of 0 and $1 / 2$.

Let us further denote the period of solution $x(t)$ as $T$. By analogy with the Mathieu equation, let us denote the proper functions that are Gegenbauer polynomes as $C_{4 j}(z)$ (the even $T / 2$-periodic solution), $C_{4 j+1}(z)$ (the odd $T$-periodic solution), $S_{4 j+2}(z)$ (the even $T$-periodic solution), and $S_{4 j+3}(z)$ (the odd $T / 2$-periodic solution). In the linear case, the trajectories of these solutions are the well-known Lissajou figures. We write the eigenvalues of these problems in the same order as above [21]:

$$
\begin{aligned}
\lambda_{4 j} & =j[(2 j+1)(r+1)-2], \\
\lambda_{4 j+1} & =(2 j+1)[j(r+1)+1], \\
\lambda_{4 j-2} & =(2 j+1)[(j+1)(r+1)-1], \\
\lambda_{4 j+3} & =(j+1)[(2 j+1)(r+1)+2] \quad(j=0,1,2, \ldots) .
\end{aligned}
$$


Note that so simple a form for the spectrum of a stability problem is not very often obtainable for the non-linear case.

\subsection{Homogeneous chain system}

For the sake of definiteness, let us consider a homogeneous chain system with two degrees of freedom:

$$
\begin{aligned}
& m_{1} \ddot{x}_{1}+a_{11} x_{1}^{r}+a_{12}\left(x_{1}-x_{2}\right)^{r}=0, \\
& m_{2} \ddot{x}_{2}+a_{22} x_{2}^{r}+a_{12}\left(x_{2}-x_{1}\right)^{r}=0 .
\end{aligned}
$$

An equation to determine the vibration forms is

$$
\mu=\frac{\alpha k^{r}-\varkappa k}{(1-k)^{r}(1+\varkappa k)}\left(\alpha=\frac{a_{22}}{a_{11}}, \mu=\frac{a_{12}}{a_{11}}, \varkappa=\frac{m_{2}}{m_{1}}, k=\frac{x_{2}}{x_{1}}\right)
$$

An examination of this relationship shows $[3,21]$ that for positive values of the constraint parameter $\mu$ there always exists one inphase vibration mode and one, two or three antiphase modes whose number depends on $\mu$ and the homogeneity index $r$. For negative values of the constraint parameter $\mu$ at any value of $r$ there exists one antiphase and one or three inphase vibration modes.

For the system in question the eigenvalue $i$ of the hypergeometric equation (6)

$$
i=\frac{r(1+x k)\left(\alpha k^{r}-x k\right)}{x(1-k)\left(\alpha k^{r}+1\right)} .
$$

The plots of $\eta=4 \pi^{-1} \operatorname{arctg}\left(\mu \cdot 2^{r} /(r-1)\right)$ and $\xi=\operatorname{arctg} k$ shown in Fig. 1 allow one to find the relation between the number of vibration modes and the parameters of the chain system. Curve (a) corresponds to the case of $r=3, \alpha=1$; (b) $r=7, \alpha=1$; (c) $r=3, \alpha=0$; (d) $r=3, \alpha=1,2$ (the value of $\varkappa=1$ ). Each first point of stability shift for $r=3$ is marked with a circle $O$. and for $r=7$, with a cross $\times$. A figure shown near a point corresponds to the number of the eigenvalue $\lambda_{i}$. The ranges in which vibration modes are unstable lie between the points whose numbers are from $4 j+1$ to $4 j+2$ and from $4 j+3$ and $4 j+4$ $(j=0,1,2, \ldots)$. In the asymmetrical case $(\lambda \neq 1)$ some points of branching turn into limit points.

Figure 2 shows plots of $\mu$ versus arctg $k$ for other values of the system parameters. Curve (e) corresponds to the limiting case $r=0, x=1, \alpha=1$; (f) $r=1 / 3, x=1, \alpha=1$; (g) $0<r<1, \varkappa>\alpha, \varkappa^{r}>\alpha$; (h) $0<r<1, \varkappa<\alpha, x^{r}<\alpha$. These plots give an idea of how great the number of vibration modes is at various $r, \alpha, x$.

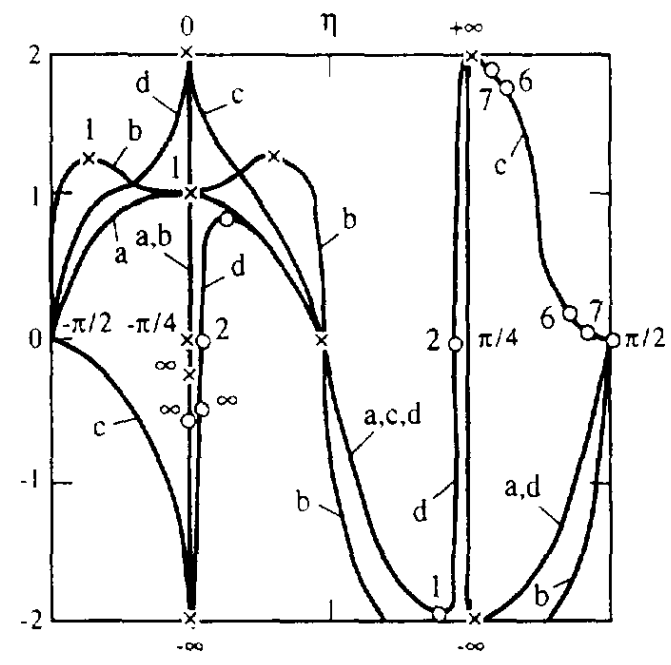

Fig. 1. The dependence between the number of vibration modes and the parameters of the system (7). Curves correspond to the cases $r=3,7 ; j=0,1,2$. Points of stability shift are marked by circles and crosses. Numbers of the eigenvalue $\lambda_{i}$ are shown near the points. 


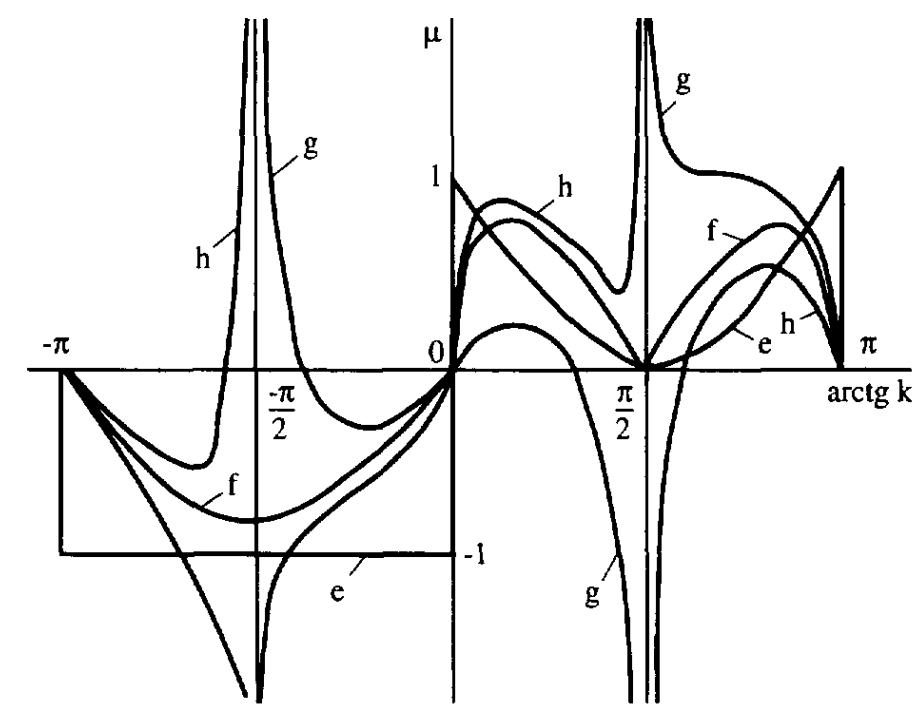

Fig. 2. The dependence between the number of vibration modes and the parameters of the system (7) correspond to $r<1$ and various values of other system parameters.

In order to find those periodic solution branching points that do not affect the stability of normal vibrations, let us write a general solution of the variational equation (6):

$$
\begin{aligned}
& v=C_{1} u_{1}(z)+C_{2} z^{1 /(r+1)} \cdot u_{5}(z) \\
&(\text { in the vicinity of the singular point } z=0), \\
& v=D_{1} u_{2}(1-z)+D_{2} \sqrt{1-z} \cdot u_{6}(1-z) \\
& \quad(\text { in the vicinity of the singular point } z=1),
\end{aligned}
$$

$C_{1}, C_{2}, D_{1}, D_{2}$ being arbitrary constants, $u_{1}(z), u_{2}(1-z), u_{5}(z), u_{6}(1-z)$ known hypergeometric functions [20]. Let us denote the matrix that relates $C_{1}, C_{2}$ to $D_{1}, D_{2}$ as $\mathbf{N}$ :

$$
\left\|C_{1}\right\|=\mathbf{N}\left\|\begin{array}{l}
D_{1} \\
C_{2}
\end{array}\right\|
$$

One can formulate denumerably many boundary problems

$$
\left(\mathbf{J N} \mathbf{N}^{-1} \mathbf{J N}\right)^{p}=\mathbf{I}(p=1,2, \ldots), \quad \mathbf{I}=\left\|\begin{array}{rr}
1 & 0 \\
0 & -1
\end{array}\right\|
$$

in order to define the branching periodic solutions traversing the singular points $z=0$ and $z=1 p$ times.

For the eigenvalue $\lambda=(r-1) / r$ such a branching periodic solution may be written in the explicit form:

$$
v=C_{1}\left(1+\sqrt{1-z^{r}}\right)^{1 / r}+C_{2} z\left(1+\sqrt{1-z^{r}}\right)^{-1 / r} .
$$

\subsection{Systems approaching homogeneity}

The results can be used for systems close to homogeneous ones. The periodic solutions separating the regions of stability and instability should be sought for the variational equations by perturbation theory. In the zeroth approximation these solutions are described by the eigenfunctions of a hypergeometric equation that correspond to the generating homogeneous system. From the conditions of the solutions periodicity, the stability shift points are determined in the further approximations.

In this section, however, only an example is given. Let us consider a symmetrical chain system whose potential contains $x_{1}$ and $x_{1}$ to the second and fourth power:

$$
\begin{aligned}
& m \ddot{x}_{1}+a_{11} x_{1}+a_{13} x_{1}^{3}+a_{21}\left(x_{1}-x_{2}\right)+a_{23}\left(x_{1}-x_{2}\right)^{3}=0, \\
& m \ddot{x}_{2}+a_{11} x_{2}+a_{23} x_{2}^{3}+a_{21}\left(x_{2}-x_{1}\right)+a_{23}\left(x_{2}-x_{1}\right)^{3}=0 .
\end{aligned}
$$


The equation that governs the anti-phase vibration mode $x_{2}=-x_{1}$ and the variational equation that permits a judgement about the orbital stability of this vibration form are as follows:

$$
\begin{gathered}
\ddot{x}+x\left(y+2 \rho x^{2}\right)=0, \\
\ddot{v}+v\left(\sigma+\beta x^{2}\right)=0, \\
\gamma=\frac{a_{11}+2 a_{21}}{m}, \quad \rho=\frac{a_{13}+8 a_{23}}{m}, \quad \sigma=\frac{a_{11}}{m}, \quad \beta=\frac{3 a_{13}}{m}, \\
x=\frac{x_{1}-x_{2}}{2}, \quad v=\frac{\delta x_{1}+\delta x_{2}}{2},
\end{gathered}
$$

or, with the primes denote the derivation with respect to $u$,

$$
v^{\prime \prime}\left[\gamma\left(1-x^{2}\right)+\rho\left(1-x^{4}\right)\right]-v^{\prime}\left(\gamma x+2 \rho x^{3}\right)+v\left(\sigma+\beta x^{2}\right)=0 .
$$

This is the Lamé equation. Let us use the perturbation theory in order to find the first two points of stability shift (corresponding to the branching of normal vibrations and periodic solutions with the period of the generating solution but displaced in phase by half-period).

Let the system be quasilinear. We assume that $\rho$ and $\beta$ are small. Let us introduce a formal small parameter $\varepsilon$ and multiply the non-linear terms by $\varepsilon$ :

$$
v^{\prime \prime}\left[\gamma\left(1-x^{2}\right)+\varepsilon \rho\left(1-x^{4}\right)\right]-v^{\prime}\left(\gamma x+2 \varepsilon \rho x^{3}\right)+v\left(\sigma+\varepsilon \beta x^{2}\right)=0 .
$$

We use the following expansions:

$$
\begin{gathered}
v=v_{0}+\varepsilon v_{1}+\cdots, \quad \gamma=\gamma_{0}+\varepsilon \gamma_{1}+\cdots, \quad \sigma=\sigma_{0}+\varepsilon \sigma_{1}+\cdots, \\
\rho=\varepsilon \rho_{1}+\varepsilon^{2} \rho_{2}+\cdots, \quad \beta=\varepsilon \beta_{1}+\varepsilon^{2} \beta_{2}+\cdots,
\end{gathered}
$$

In the zeroth approximation with respect to $\varepsilon$, one obtains

$$
v_{0}^{\prime \prime} \gamma_{0}\left(1-x^{2}\right)-v_{0}^{\prime} \gamma_{0} x+v_{0} \sigma_{0}=0 .
$$

In the first stability shift point, normal vibrations branch: $v_{0}=k x$ (here $x=\cos \omega t$ ) and $\gamma_{0}=\sigma_{0}$. Therefore, in the zeroth approximation the linear system is uncoupled, that is $a_{21}=0$. We may readily check that it is at this point that the periodic solutions with the period of the generating solution which are displaced in phase by a half-period branch too: $v_{0}=k \dot{x}_{0}$ (here $\dot{x}_{0}=\sin \omega t, \dot{x}_{0}=\sqrt{1-x^{2}}$ ). Consequently, in the zeroth approximation the equality $\gamma_{0}=\sigma_{0}$ holds for the second stability shift point as well.

Let us write the first approximation with respect to $\varepsilon$ :

$$
v_{1}^{\prime \prime} \gamma_{0}\left(1-x^{2}\right)-v_{1}^{\prime} \gamma_{0} x+v_{1} \sigma_{0}-v_{0}^{\prime}\left(\gamma_{1} x+2 \rho_{1} x^{3}\right)+v_{0}\left(\sigma_{1}+\beta_{1} x^{2}\right)=0
$$

(for the first point), or

$$
\gamma_{0}\left[v_{1}^{\prime \prime}\left(1-x^{2}\right)-v_{1}^{\prime} x+v_{1}\right]=k\left[\left(\gamma_{1}-\sigma_{1}\right) x+\left(2 \rho_{1}-\beta_{1}\right) x^{3}\right] .
$$

The periodicity conditions are the conditions of orthogonality of the right-hand side with respect to the periodic solution of the homogeneous equation. Denoting a line integral along a closed contour (along the period) by $\oint$, one obtains for the first stability shift point

$$
\oint\left[\left(\gamma_{1}-\sigma_{1}\right) x^{2}+\left(2 \rho_{1}-\beta_{1}\right) x^{4}\right] \mathrm{d} t=0
$$

or

$$
\left(\gamma_{1}-\sigma_{1}\right) \oint \frac{x^{2} \mathrm{~d} x}{\sqrt{1-x^{2}}}+\left(2 \rho_{1}-\beta_{1}\right) \oint \frac{x^{4} \mathrm{~d} x}{\sqrt{1-x^{2}}}=0 .
$$

After simple calculations one finds that

$$
\frac{\left(\gamma_{1}-\sigma_{1}\right)}{\left(\beta_{1}-2 \rho_{1}\right)}=\frac{3}{4}
$$


For the second stability shift point, the periodicity condition is

$$
\oint\left[v_{0}^{\prime}\left(\gamma_{1} x+2 \rho_{1} x^{3}\right)-v_{0}\left(\sigma_{1}+\beta_{1} x^{2}\right)\right] \dot{x} \mathrm{~d} t=0
$$

Here $v_{0}=C \dot{x}=C \sin \omega t, x=\cos \omega t$. After simple rearrangements one obtains

$$
\frac{\left(\gamma_{1}-\sigma_{1}\right)}{\left(\beta_{1}-6 \rho_{1}\right)}=\frac{1}{4}
$$

Let us now consider a quasihomogeneous system. Assume that $\gamma$ and $\sigma$ are small. We introduce a formal small parameter $\varepsilon$ before each linear term:

$$
v^{\prime}\left[\varepsilon \gamma\left(1-x^{2}\right)+\rho\left(1-x^{4}\right)\right]-v^{\prime}\left(\varepsilon \gamma x+2 \rho x^{3}\right)+v\left(\varepsilon \sigma+\beta x^{2}\right)=0 .
$$

We use the following expansions:

$$
\begin{gathered}
v=v_{0}+\varepsilon v_{1}+\cdots, \quad \gamma=\varepsilon \gamma_{1}+\cdots, \quad \sigma=\varepsilon \sigma_{1}+\cdots, \\
\rho=\rho_{0}+\varepsilon \rho_{1}+\cdots, \quad \beta=\beta_{0}+\varepsilon \beta_{1}+\cdots .
\end{gathered}
$$

In the zeroth approximation with respect to $\varepsilon$

$$
v_{0}^{\prime \prime} \rho_{0}\left(1-x^{4}\right)-v_{0}^{\prime} \cdot 2 \rho x_{0}^{3}+v_{0} \cdot \beta x^{2}=0
$$

The branching of normal vibrations (the first stability shift point) $v_{0}=C x$ provided that $\beta_{0}=2 \rho_{0}$. For the second point, that is the branching point of solutions $v_{0}=C \dot{x}$ the conditions will be $\beta_{0}=6 \rho_{0}$.

In the first approximation with respect to $\varepsilon$ we write

$$
\begin{gathered}
v_{1}^{\prime \prime} \rho_{0}\left(1-x^{4}\right)-v_{1}^{\prime} \rho_{0} x^{3}+v_{1} \beta x^{2}-v_{0}^{\prime \prime}\left[\rho_{1}\left(1-x^{4}\right)+\gamma_{1}\left(1-x^{2}\right)\right] \\
-v_{0}^{\prime}\left(2 \rho_{1} x^{3}+\gamma_{1} x\right)+v_{0}\left(\beta_{1} x^{2}+\sigma_{1}\right)=0 .
\end{gathered}
$$

From the periodicity conditions for the first and second points of stability shift we obtain, respectively:

$$
\begin{aligned}
& \oint\left[x\left(2 \rho_{1} x^{3}+\gamma_{1} x\right)-x\left(\beta_{1} x^{2}+\sigma_{1}\right) x\right] \mathrm{d} t=0 . \\
& \oint\left[\dot{x}\left(2 \rho_{1} x^{3}+\gamma_{1} x\right)-\dot{x}\left(\beta_{1} x^{2}+\sigma_{1}\right) \dot{x}\right] \mathrm{d} t=0 .
\end{aligned}
$$

Upon rearrangement we have

$$
\frac{\left(\gamma_{1}-\sigma_{1}\right)}{\left(\beta_{1}-2 \rho_{1}\right)}=\frac{1}{12}\left[\frac{\Gamma(1 / 4)}{\Gamma(3 / 4)}\right]^{2} \approx 0.729
$$

for the first point and

$$
\frac{\left(\gamma_{1}-\sigma_{1}\right)}{\left(\beta_{1}-6 \rho\right)}=\frac{12}{5}\left[\frac{\Gamma(3 / 4)}{\Gamma(1 / 4)}\right]^{2} \approx 0.274
$$

for the second point, of stability shift.

\section{FINITE ZONING CONDITIONS FOR A STABILITY PROBLEM BY THE FIRST APPROXIMATION}

\subsection{Finite number of orbital stability zones}

Let us consider a situation when the number of orbital stability zones of normal vibration modes is finite (it is about a first approximation of stability and instability). Then all other instability zones are contracted to lines ("collapse"). This situation may be realized under certain conditions imposed upon the systems parameters. If these conditions are not satisfied, the number of instability zones will obviously be infinite (e.g. as on the well known Ince-Strutt diagram for the Mathieu equation). However, the importance of finite zoning 
conditions (or finite-gap conditions) depends on the fact that all the instability zones, except a finite number of them, are "narrow" if the values of the parameters are "close" to values which guarantee a realization of the finite zoning conditions when these zones are contracted to lines. It is interesting that sometimes the first parametric resonance zone may be "narrow" in this sense though it is usually considered as wide (e.g. for the Mathieu equation). Moreover, the finite zoning conditions are of interest for the problem of solitons in non-linear wave systems.

So, let us consider a conservative system having two degrees of freedom, which may be described by the following equations

$$
\ddot{x}_{i}+\frac{\partial \Pi}{\partial x_{i}}=0 \quad(i=1,2)
$$

where $\Pi$ is the analytically positively determined potential energy.

One may assume the system to permit rectilinear normal vibration modes $x_{2}=k x_{1}$. Let us separate one of the modes and reduce it to $x_{2}=0$ by rotation of the coordinate axes. Then

$$
\Pi\left(x_{1}, x_{2}\right)=\sum_{i=2}^{m} a_{i} x_{1}^{i}+x_{2}^{2} \sum_{i=0}^{m-2} e_{i} x_{1}^{i}+\sum_{i=3}^{\infty} x_{2}^{i} g_{i}\left(x_{1}\right),
$$

where $g_{i}\left(x_{1}\right)$ are analytical functions. The condition of existence of the solution $x_{2}=0$ is obviously satisfied.

In the normal vibration mode the system behaves like a system with one degree of freedom

$$
\ddot{x}+\Pi_{x_{1}}(x, 0)=0 \quad\left(x \equiv x_{1}\right)
$$

with the energy integral

$$
\frac{\dot{x}}{2}+\Pi(x, 0)=h
$$

The orbital stability of normal mode $x_{2}=0$ is related to $y$ variations orthogonal to the trajectory. The variational equation is:

$$
\ddot{y}+y \Pi_{x_{2} x_{2}}(x, 0)=0 .
$$

The function $\Pi_{x_{2} x_{2}}(x(t), 0)$ is polynomial with respect to $x$ and periodical with respect to $t$.

The finite zoning criterion for equations as (15) was given in $[22,23]$ by Novikov. Namely, periodic potentials $u(t)$ of the Schrödinger equation

$$
\ddot{\psi}+[e-u(t)] \psi=0
$$

may have just $n$ finite restricted zones (instability zones) if the equation

$$
i \frac{\mathrm{d} x}{\mathrm{~d} t}-x^{2}=u-e
$$

admits of a solution

$$
\varkappa=\frac{D-(1 / 2) \dot{z}}{z}
$$

where $z$ is a polynomial in $e$ of degree $n$ with variable factors $\left(z=\sum_{k=0}^{n} \hat{x}_{k}(t) e^{k}\right), D$ being a constant. Then the equation for the $z$ polynomial may be written down as:

$$
\ddot{z}-4(u-e) \dot{z}-2 \dot{u} z=0 .
$$

Comparing (15) and (16) one may conclude that $u-e=-\Pi_{x_{1} x_{2}}(x, 0)$. Taking into consideration the initial expression (12) for potential energy $\Pi\left(x_{1}, x_{2}\right)$, we have $e=2 e_{0}$.

\subsection{Finite zoning conditions}

Let us consider an algebraic form of the equation (17) (algebraization in the Ince sense [14]) to obtain finite zoning conditions as some conditions imposed on factors of potential 
energy $\Pi\left(x_{1}, x_{2}\right)$ expansion. Namely, let us introduce a new independent variable $x$ instead of $t$. Taking the following equalities into account

$$
\begin{gathered}
\frac{\mathrm{d}}{\mathrm{d} t}=\frac{\mathrm{d}}{\mathrm{d} x} \dot{x}, \quad \frac{\mathrm{d}^{2}}{\mathrm{~d} t^{2}}=\frac{\mathrm{d}^{2}}{\mathrm{~d} x^{2}} \dot{x}^{2}+\frac{\mathrm{d}}{\mathrm{d} x} \ddot{x}, \quad \frac{\mathrm{d}^{3}}{\mathrm{~d} t^{3}}=\frac{\mathrm{d}^{3}}{\mathrm{~d} x^{3}} \dot{x}^{3}+3 \frac{\mathrm{d}^{2}}{\mathrm{~d} x^{2}} \dot{x} \ddot{x}+\frac{\mathrm{d}}{\mathrm{d} x} \dddot{x}, \\
\dot{x}^{2}=2(h-\Pi(x, 0)), \quad \ddot{x}=-\Pi_{x}(x, 0), \quad \dddot{x}=-\Pi_{x}(x, 0) \dot{x}
\end{gathered}
$$

[the last three equalities were obtained from (13) and (14)]. One may have an equation with regular singularities instead of equation (17) with periodic coefficients:

$$
\begin{gathered}
2 z^{\prime \prime \prime}(h-\Pi(x, 0))+3 z^{\prime \prime}\left(-\Pi_{x}(x, 0)\right)+z^{\prime}\left[-\Pi_{x x}(x, 0)+4 \Pi_{x_{2} x_{2}}(x, 0)\right] \\
+2 z \frac{\partial}{\partial x}\left[\Pi_{x_{2} x_{2}}(x, 0)\right]=0
\end{gathered}
$$

where a prime symbol denotes differentiation with respect to $x$.

Then the $n$-zoning condition means the solution of equation (18) to be a polynomial in $e$, the coefficients being functions of $x$ :

$$
z=\sum_{k=0}^{n} \alpha_{k}(x) e^{k}
$$

Substituting (19) into (18) and grouping the terms with equal powers of $e$, one may have a problem in the eigenvalues $a_{i}, e_{i}$.

\subsection{Characteristic classes of analytical potential}

This problem is absolutely boundless in general, so let us describe results for three characteristic classes of analytical potentials. Moreover, we should confine ourselves to the values $n=0,1,2$.

4.3.1. $a_{2} \neq 0, a_{4} \neq 0, e_{0} \neq 0, e_{2} \neq 0$, the rest of the factors in (12) being zero. Note, that equation (11) in this case has linear and cubic terms with respect to $x_{1}, x_{2}$.

Grouping the terms with identical powers of $e$, then grouping the terms with identical powers of $x$, we may obtain the finite zoning condition:

$$
e_{2}=(n+1) n a_{4}
$$

As shown in this section, the equation in variations is reduced to the Lame equation in this case, and the finite zoning conditions are known $[11,17]$. Hence, let us discuss other potentials for which finite zoning conditions were determined for the first time in [24].

4.3.2. $a_{2} \neq 0, a_{4} \neq 0, a_{6} \neq 0, e_{0} \neq 0, e_{2} \neq 0, e_{4} \neq 0$, the rest of the factors in (12) being zero. Equation (11) in this case contains the terms of the first, third and fifth powers of $x_{1}, x_{2}$.

Grouping the terms with identical powers of $e$, then grouping the terms with identical powers of $x$, we may obtain the finite zoning conditions:

$$
e_{4}=4(n+1) n a_{6} ; \quad e_{2}=2(n+1) n a_{4} ; \quad 4 a_{2} a_{4} a_{6}-a_{4}^{3}+8 h a_{6}^{2}=0
$$

4.3.3. $a_{2} \neq 0, a_{3} \neq 0, a_{4} \neq 0, e_{0} \neq 0, e_{1} \neq 0, e_{2} \neq 0$, the rest of the factors in (12) being zero. Equation (11) in this case contains the terms of the first, second and third powers of $x_{1}, x_{2}$.

Grouping the terms with identical powers of $e$, then grouping the terms with identical powers of $x$, we may obtain the finite zoning conditions:

$$
e_{2}=(n+1) n a_{4} ; \quad e_{1}=\frac{1}{2}(n+1) n a_{3} ; \quad 4 a_{2} a_{4}=a_{3}^{2} .
$$

The finite zoning conditions (20) for case 4.3.1 is valid for any positive integer $n$, but conditions (21) for the case 4.3.2 and (22) for the case 4.3.3 were obtained only for $n=0,1,2$. One may repeat all the calculations for $n>2$ selecting the terms with senior powers of $x$ to realize that the first two equations in (21) and (22) are valid again. 


\subsection{Eigenfunctions and eigenvalues}

Now let us discuss the calculation of eigenfunctions and eigenvalues for a finite zoning case. At the same time conditions (20)-(22) will be obtained in another way for any $n$.

Let us transform the variational equation (15) to an algebraic form introducing a new independent variable $x$ instead of $t$. Then equation (15) will be

$$
2 y^{\prime \prime}(h-\Pi(x, 0))+y^{\prime}\left(-\Pi_{x}(x, 0)\right)+y \Pi_{x_{2} x_{2}}(x, 0)=0 .
$$

This is an equation of Fuchs class for the polynomial potentials given above, whose exponents of the finite singularities equal 0 or $1 / 2$ (the singularities here are zeros of the expression $h-\Pi(x, 0)$ and $x=\infty)$. Hence, one may search all the eigenfunctions as (the number of the eigenfunctions are $2 n+1$ for the case of $n$-zoning):

$$
y=p(x) \quad \text { or } \quad y=\sqrt{x-\xi} R(x)
$$

where $p(x)$ and $R(x)$ are analytical functions and $x=\xi$ is a singular point of the equation. Indeed, that way one may find all the eigenfunctions and eigenvalues for the potentials I, II, III when $n=0,1,2$.

Let us consider another approach. First, let us write down the variational equations in the algebraic form for the three types of potentials:

$$
\begin{aligned}
& \text { I: } \quad 2 y^{\prime \prime}\left(h-a_{2} x^{2}-a_{4} x^{4}\right)+y^{\prime}\left(-2 a_{2} x-4 a_{4} x^{3}\right)+y\left(e+2 e_{2} x^{2}\right)=0 \\
& \text { II: } \quad 2 y^{\prime \prime}\left(h-a_{2} x^{2}-a_{4} x^{4}-a_{6} x^{6}\right)+y^{\prime}\left(-2 a_{2} x-4 a_{4} x^{3}-6 a_{6} x^{5}\right) \\
& \quad+y\left(e+2 e_{2} x^{2}+2 e_{4} x^{4}\right)=0
\end{aligned}
$$

III: $\quad 2 y^{\prime \prime}\left(h-a_{2} x^{2}-a_{3} x^{3}-a_{4} x^{4}\right)+y^{\prime}\left(-2 a_{2} x-3 a_{3} x^{2}-4 a_{4} x^{3}\right)$

$$
+y\left(e+2 e_{1} x+2 e_{2} x^{2}\right)=0
$$

Then let us write the known Lamé equation in an algebraic form

$$
y^{\prime \prime}\left(z^{2}-a^{2}\right)\left(z^{2}-b^{2}\right)+y^{\prime} z\left(2 z^{2}-a^{2}-b^{2}\right)-y\left[n(n+1) z^{2}-\lambda\right]=0
$$

(finite zoning for $n=0,1,2,3, \ldots$ ).

Equation (26) coincides with (23) if

$$
x=z, \quad h / a_{4}=-a^{2} b^{2}, \quad a_{2} / a_{4}=-a^{2}-b^{2}, \quad e_{0} / a_{4}=-i, \quad e_{2} / a_{4}=n(n+1)
$$

(the last equality is the finite zoning condition for $n=0,1,2, \ldots$ ). Connecting (26) and (23), one may note that the coefficient of $y^{\prime \prime}$ in (26) has the meaning of the kinetic energy $(h-\Pi(x, 0))$ for periodic motion $x(t)$. Kinetic energy vanishes twice per period at the amplitude values. Without loss of generality one may assume $x= \pm a$. Then one may assume $a>0,|z| \leqslant a$ and either $b^{2}<0$ or $b^{2}>a^{2}$.

All the eigenfunctions and eigenvalues of the Lamé equation for $n=0,1,2$ may be easily found by substitution into (26) of polynomials or polynomials multiplied by $\sqrt{a^{2}-z^{2}}$, $\sqrt{z^{2}-b^{2}}$ or $\sqrt{b^{2}-z^{2}}$. Exactly such functions have time period $T$ and $T / 2(T$ being a period of a function $z=x(t))$ and define the bounds of stability or instability zones according to the Floques-Lyapunov theory. The following eigenfunctions $y$ and eigenvalues $\lambda$ may be obtained (eigenfunctions being written down in succession according to increase of the number of zeros; $C$ being an arbitrary constant):

$n=0$ :

$$
r_{0}=C, \quad i_{0}=0
$$

$n=1\left(b^{2}<0\right):$

$$
\begin{array}{ll}
y_{0}=C \sqrt{z^{2}-b^{2}}, & \lambda_{0}=a^{2}, \\
y_{1}=C z, & \lambda_{1}=a^{2}+b^{2}, \\
y_{2}=C \sqrt{a^{2}-z^{2}}, & \lambda_{2}=b^{2},
\end{array}
$$


$n=1\left(b^{2}>a^{2}\right)$

$$
\begin{array}{ll}
y_{0}=C \sqrt{b^{2}-z^{2}}, & \lambda_{0}=a^{2}, \\
y_{1}=C \sqrt{a^{2}-z^{2}}, & \lambda_{1}=b^{2}, \\
y_{2}=C z, & \lambda_{2}=a^{2}+b^{2},
\end{array}
$$

$n=2\left(b^{2}<0\right)$

$$
\begin{array}{ll}
y_{0}=C\left(z^{2}-\frac{2 a^{2} b^{2}}{\lambda_{0}}\right), & \lambda_{0}=2\left[a^{2}+b^{2}+\sqrt{\left(a^{2}+b^{2}\right)^{2}-3 a^{2} b^{2}}\right], \\
y_{1}=C z \sqrt{z^{2}-b^{2}}, & \lambda_{1}=4 a^{2}+b^{2}, \\
y_{2}=C \sqrt{z^{2}-b^{2}} \sqrt{a^{2}-z^{2}}, & \lambda_{2}=a^{2}+b^{2}, \\
y_{3}=C z \sqrt{a^{2}-z^{2}}, & \lambda_{3}=a^{2}+4 b^{2}, \\
y_{4}=C\left(z^{2}-\frac{2 a^{2} b^{2}}{\lambda_{4}}\right), & \lambda_{4}=2\left[a^{2}+b^{2}-\sqrt{\left(a^{2}+b^{2}\right)^{2}-3 a^{2} b^{2}}\right]
\end{array}
$$

$n=2\left(b^{2}>a^{2}\right)$

$$
\begin{array}{ll}
y_{0}=C\left(z^{2}-\frac{2 a^{2} b^{2}}{\lambda_{0}}\right), & \lambda_{0}=2\left[a^{2}+b^{2}-\sqrt{\left(a^{2}+b^{2}\right)^{2}-3 a^{2} b^{2}}\right] \\
y_{1}=C \sqrt{b^{2}-z^{2}} \sqrt{a^{2}-z^{2}}, & \lambda_{1}=a^{2}+b^{2}, \\
y_{2}=C z \sqrt{a^{2}-z^{2}}, & \lambda_{2}=4 a^{2}+b^{2}, \\
y_{3}=C z \sqrt{a^{2}-z^{2}}, & \lambda_{3}=a^{2}+4 b^{2}, \\
y_{4}=C\left(z^{2}-\frac{2 a^{2} b^{2}}{\lambda_{4}}\right), & \lambda_{4}=2\left[a^{2}+b^{2}+\sqrt{\left(a^{2}+b^{2}\right)^{2}-3 a^{2} b^{2}}\right] .
\end{array}
$$

The following intervals of the $\lambda$ axis correspond to instability zones: $\left(-\infty, \lambda_{0}\right]$, $\left[\lambda_{1}, \lambda_{2}\right],\left[\lambda_{3}, \lambda_{4}\right]$. Figures 3 and 4 present a stability chart of the Lamé equation for $n=3$ and $n \neq 3$ but "close" to 3 .

Other variational equations may be also be reduced to the Lamé equation (26). Equation (25) may be reduced to the Lamé equation by shifting along the real axis (the number of singularities being constant and their symmetry about the origin of coordinates being restored)

$$
\begin{gathered}
z=x+\mu, \quad \text { where } \mu= \pm \sqrt{\left(a^{2}+b^{2}\right) / 2} \\
\text { if } h / a_{4}=\frac{1}{4}\left(a^{2}-b^{2}\right)^{2}, \quad a_{2} / a_{4}=2\left(a^{2}+b^{2}\right), \quad a_{3} / a_{4}=4 \mu \\
e_{0} / a_{4}=-\lambda+\mu^{2} n(n+1), \quad e_{1} / a_{4}=2 \mu n(n+1), \quad e_{2} / a_{4}=n(n+1) .
\end{gathered}
$$

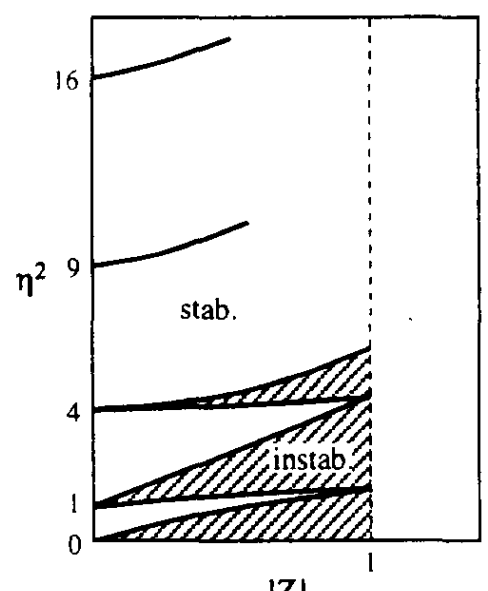

$|Z|$

Fig. 3. Stability chart of the Lamé equation for $n=3$ (finite zoning case). 


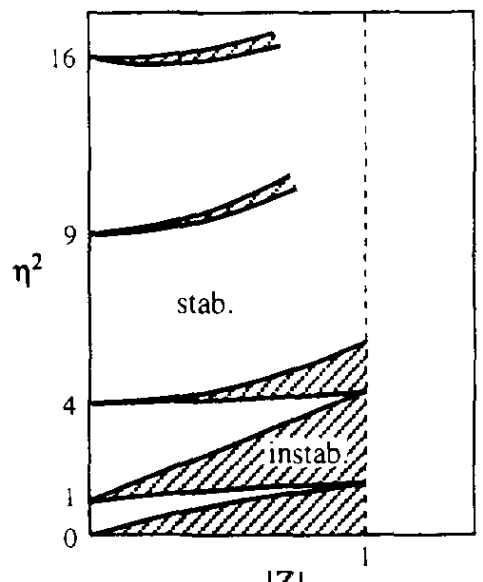

$|Z|$

Fig. 4. Stability chart of the Lamé equation if the parameter values close to values which guarantee a realization of the finite zoning conditions.
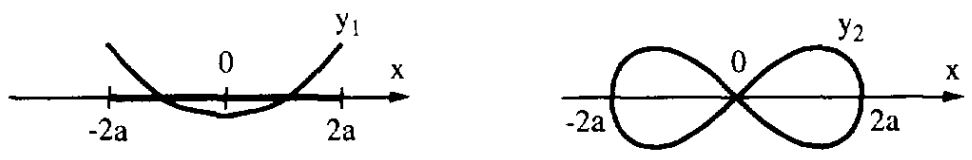

Fig. 5. Eigenfunctions of the variational equation (24) corresponding to the boundaries of the finite zone of instability $\left(n=1, b^{2}<0\right)$.

Note, that the finite zoning conditions (22) for any positive integer $n$ are the consequences of these expressions. This shift makes it possible to find all eigenfunctions and eigenvalues for the equation (25) from the functions obtained for the Lamé equation.

Equation (24) may be reduced to the Lamé equation by a quadratic law transformation $z=x^{2} a$ if (then the number of singularities being changed)

$$
\begin{array}{cl}
h / a_{6}=2 a\left(a^{2}-b^{2}\right), & a_{2} / a_{6}=5 a^{2}-b^{2}, \quad a_{4} / a_{6}=-4 a, \\
e_{6} / a_{6}=4\left[a^{2} n(n+1)-\lambda\right], & e_{2} / a_{6}=-8 a n(n+1), \quad e_{4} / a_{6}=4 n(n+1) .
\end{array}
$$

Hence the finite zoning conditions (21) for any positive integer $n$ may be obtained. The eigenfunctions and eigenvalues are transformed accordingly.

It is remarkable that the first zone of parametric resonance is being contracted to a line in the case II when the finite zoning conditions are valid. Indeed, the eigenfunctions of equation (24) corresponding to the boundaries of the finite zone of instability, e.g. for $n=1$ $\left(b^{2}<0\right)$, are (Fig. 5)

$$
y_{1}=C\left(x^{2}-a\right), \quad y_{2}=C \sqrt{a^{2}-\left(x^{2}-a\right)^{2}} .
$$

For a periodic mode: $-2 a \leqslant x \leqslant 2 a$. But the functions $y_{1}(x)$ and $y_{2}(x)$ turn to zero within the interval of $[-2 a, 2 a]$, i.e. their period with respect to $t$ is $T / 2$ ( $T$ being the period of $x(t), x$ being changed within these limit (on a half of the period). Functions corresponding to the first zone of parametric resonance should have the period of $T$ ).

If conditions (20)-(22) are not strictly met, the number of instability zones will be infinite. However all of them are "narrow" in the sense mentioned except the selected $n$ zones, since the zones are contracted to lines when the finite zoning conditions are valid. This is also true for the first zone of parametric resonance which is usually assumed to be "wide".

Note once again that we will obtain the finite zoning conditions (20)-(22) for an arbitrary positive integer $n$ transforming the Lamé equation into variational equations as mentioned above (parameters being changed continuously).

\subsection{Pendulum systems}

Let us consider another class of non-linear systems-- pendulum systems with the potential

$$
V=-a \cos x_{1}+x_{2}^{2}(b+d \cos x)+x_{2}^{3} g\left(x_{1}, x_{2}\right)
$$


Linearization around the normal mode of vibration $x_{2}=0, x_{1}=x(t)$ (the equation of motion $\ddot{x}+a \sin x=0)$ gives the variational equation $\ddot{y}+2(b+d \cos x) y=0$. In this case a solution $x(t)$ may be either periodic or aperiodic (oscillations and rotation of the pendulum). Introducing a new independent variable $z=\dot{x}^{2} / 2$, one may transform the variational equation to:

$$
y^{\prime \prime}+\frac{1}{2} y^{\prime}\left[\frac{1}{z}+\frac{1}{z-(h+a)}+\frac{1}{z-(h-a)}\right]+y \frac{-2 b-\frac{2 b}{a}(z-h)}{4 z[z-(h+a)][z-(h-a)]}=0
$$

being the Lamé equation [17]. Here the finite zoning conditions are:

$$
\frac{2 d}{a}=n(n+1) \quad(n=0,1,2, \ldots)
$$

( $n$ being a number of finite zones of instability within the space of system parameters).

\subsection{General procedure of transformation}

Let us describe the general procedure of transformation from the finite zoning Lamé equation (26) to the variational equation (15) in an algebraic form. One should multiply (26) by an arbitrary function $q(z)$ and perform the transformation $z=f(x)$. Comparing the equation obtained with equation (15), one should have the following conditions:

$$
\begin{gathered}
2(h-\Pi(x, 0))\left(f^{\prime}\right)^{2}=\left(f^{2}-a^{2}\right)\left(f^{2}-b^{2}\right) q(f), \\
2(h-\Pi(x, 0)) f^{\prime \prime}-\Pi_{x}(x, 0) f^{\prime}=f\left(2 f^{2}-a^{2}-b^{2}\right) q(f), \\
\Pi_{x_{2} x_{2}}(x, 0)=-\left[n(n+1) f^{2}-\lambda\right] q(f) \quad(n=0,1,2 \ldots) .
\end{gathered}
$$

Excluding the function $q(f)=\frac{\Pi_{x_{2} x_{2}}(x, 0)}{\lambda-n(n+1) f^{2}}$, one would have

$$
\begin{gathered}
2(h-\Pi(x, 0))\left[\lambda-n(n+1) f^{2}\right]\left(f^{\prime}\right)^{2}=\Pi_{x_{2} x_{2}}(x, 0)\left(f^{2}-a^{2}\right)\left(f^{2}-b^{2}\right), \\
{\left[2(h-\Pi(x, 0)) f^{\prime \prime}-\Pi_{x}(x, 0) f^{\prime}\right]\left[\lambda-n(n+1) f^{2}\right]=f\left(2 f^{2}-a^{2}-b^{2}\right) \Pi_{x_{2} x_{2}}(x, 0) .}
\end{gathered}
$$

These equations may be used to solve the following problem. Let us assume the transformation $z=f(x)$. Then one should select the value of potential energy $\Pi\left(x_{1}, x_{2}\right)$ to satisfy the equalities. The conditions imposed upon the coefficients of potential energy are the finite zoning conditions for a positive integer $n$.

Note that stability of some modes of oscillations has been investigated in [12] for a particle on a plane. In many cases the stability problem may be reduced to analysis of the finite zoning Lamé equation.

The results presented in this paragraph are not obvious. Suffice it to mention the possibility of the first zone of parametric resonance being contracted to a line.

\section{NON-LINEAR STABILITY OF NORMAL VIBRATIONS}

A problem of non-linear stability of normal vibrations in conservative systems may be solved only after the corresponding linear problem has been solved. The non-linear analysis is applicable to a system class, such that the variational equations are reduced to hypergeometric equations or Lamé equations, etc. The problem of non-linear stability of normal vibrations was also discussed by Pecelli and Thomas [11,12] (using an approach first published by Arnol'd and Mozer) for a system with two degrees of freedom and a Hamiltonian

$$
H=\frac{1}{2}\left(y_{1}^{2}+y_{2}^{2}\right)+\Pi\left(x_{1}, x_{2}\right),
$$

$x_{1}, x_{2}$ being positional variables, $y_{1}, y_{2}$ the corresponding impulses. Assume that the system allows the normal form of vibrations $x_{2}=y_{2}=0$.

What we have in view is the isoenergetic stability of the vibration form. Introducing, following [11], the variables $R, \tau, x, y$ by a substitution

$$
y_{1}=\sqrt{2 R} \sin \tau, \quad x_{1}=\sqrt{2 R} \cos \tau, \quad x_{2}=x, \quad y_{2}=y,
$$

one obtains $R(x, y)$ from the equation $H=h$. 
If $\tau$ is a new independent variable, one obtains [25]

$$
\frac{\mathrm{d} x}{\mathrm{~d} \tau}=\frac{\partial R}{\partial y}, \quad \frac{\mathrm{d} y}{\mathrm{~d} \tau}=-\frac{\partial R}{\partial x}
$$

Setting $\varkappa(0)=\varkappa_{1}, y(0)=x_{2}$, expansions

$$
x=\sum_{i, j=0}^{\infty} a_{i j}(\tau) \varkappa_{1}^{i} \varkappa_{2}^{j}, \quad y=\sum_{i, j=0}^{\infty} b_{i j}(\tau) \varkappa_{1}^{i} \varkappa_{2}^{j},
$$

may be constructed. On substituting these series into (30), one is able to find the coefficients $a_{i j}(\tau), b_{i j}(\tau)$. In this case, $a_{10}, a_{01}, b_{10}, b_{01}$ are solutions of the linear variational equation

$$
\dot{Z}=A Z
$$

wherein

$$
Z=\left\|\begin{array}{l}
x \\
y
\end{array}\right\|
$$

The series (31) define the solution $P(\tau)$ of $(30)$. What is known as Poincare transformation is $P(2 \pi)$. Thus comparison is made between the values of $x(0), y(0)$ and $x(2 \pi), y(2 \pi)$. Stability is ensured when the linearized Poincare transformation $P_{1}(2 \pi)$ defined by the coefficients $a_{10}, a_{01}, b_{10}, b_{01}$ has eigenvalues $\lambda_{ \pm}$with a modulus equal to unity and when the condition $\lambda^{k} \neq 1$ holds for $k=1,2,3,4[26,27]$.

The eigenvalue problem

$$
\left|\begin{array}{ll}
a_{10}(2 \pi)-\lambda & a_{01}(2 \pi) \\
b_{10}(2 \pi) & b_{01}(2 \pi)-i
\end{array}\right|=0
$$

implies that $\lambda=\Delta \pm \sqrt{\Delta^{2}-4 / 2}$, where $\Delta=\left(a_{10}(2 \pi)+b_{01}(2 \pi)\right)$. It is assumed here that the solutions of the homogeneous equations (32) are so normalized that $a_{01}(2 \pi) b_{10}(2 \pi)=1$.

The above limitations on the eigenvalues are satisfied with $\Delta^{2}<4, \Delta \neq 0,-1$. Under these conditions, a change of variable $(x, y)=S(\xi, \eta)$ is possible that reduces the Poincare transformation to what is known as the Birkhoff normal form $[26,27], N=S^{1} P S$. By introducing the complex variable $\zeta=\xi+$ i $\eta$, one obtains, instead of $P(2 \pi)$, the transformation $\zeta^{\prime}=\lambda \zeta \mathrm{e}^{\mathrm{i} y \zeta \zeta}+\mathrm{O}_{4}$ where $\mathrm{O}_{4}$ refers to the terms of the fourth-order with respect to $\zeta$ and $\bar{\zeta}$.

If we now consider normal vibrations that are stable in the first approximation (which implies that $\Delta^{2}<4$, for in this case the eigenvalues of the linearized transformation have moduli equal to unity) and assume $\Delta \neq 0,-1$ and $\gamma \neq 0$, it follows from the ArnoldMozer-Ruisman theorem $[26,27]$ that the normal form of vibrations possesses orbital stability in a $2 n$-dimensional phase space. It is evident that in the system parameter space, the stability regions $\Delta=0, \Delta=-1$ and $\gamma=0$ have a smaller dimension than the instability region $\Delta^{2} \geqslant 4$.

Once again it is highlighted that it is only the knowledge of the solution of the linearized variational equation that is necessary to calculate the values of $\Delta$ and $\gamma$ that define the instability regions obtained when taking into account non-linear terms.

This section deals with isoenergetic stability (for fixed values of the energy $h$ ) of normal vibrations; however, it was shown [26] that complete stability takes place when $R=\Phi(x, y)$ is continuously dependent on the energy $h$.

More detailed analysis to a specific Hamiltonian of the type

$$
H=\frac{1}{2}\left(y_{1}^{2}+y_{2}^{2}\right)-a_{2} x_{1} x_{2}+\frac{1}{2}\left(a_{1}+a_{2}\right)\left(x_{1}^{2}+x_{2}^{2}\right)+\frac{b_{1}}{4}\left(x_{1}^{4}+x_{2}^{4}\right)+\frac{b_{2}}{4}\left(x_{1}-x_{2}\right)^{4}
$$

is given in [11].

The problem of non-linear stability of normal vibrations was also discussed by Month and Rand [8]. 


\section{REFERENCES}

1. R. M. Rosenberg, The normal modes of nonlinear n-degree-of freedom systems. J. Appl. Mech. 30, 7 (1962).

2. R. M. Rosenberg, Nonlinear vibrations of systems with many degrees of freedom. Adv. Appl. Mech. 9, 156 (1966).

3. L. I. Manevich, Yu. V. Mikhlin and V. N. Pilipchuk, The Method of Normal Oscillation for Essentially nonlinear Systems. Nauka, Moscow (1989). [In Russian.]

4. A. F. Vakakis, Non-similar normal oscillations in a strongly non-linear discrete system. J. Sound Vibr. 158, 341 (1992).

5. R. M. Rosenberg and C. S. Hsu, On the geometristion of normal vibrations of nonlinear systems having many degree of freedom. In Proc. Int. Symp. Theory of Non-Linear Vibrations, Kiev, 1961. Academy of Sciences, Kiev, Vol. 1, pp. 380-416 (1963).

6. C. P. Atkinson, S. J. Bhatt and T. Pacitti, The stability of the normal modes of nonlinear systems with polynomial restoring forces of high degree. J. Appl. Mech. 31, 193 (1963).

7. L. A. Month and R. Rand, The stability of bifurcating periodic solutions in a two-degree-of-freedom nonlinear system. J. Appl. Mech. 44, 782 (1977).

8. L. A. Month and R. Rand, An application of the Poincare map to the stability of nonlinear normal modes. J. Appl. Mech. 47, 645 (1980).

9. C. H. Pac, On the stability behavior of bifurcated normal modes in coupled nonlinear systems. J. Appl. Mech. 56, $155(1989)$.

10. R. Rand, The geometrical stability of nonlinear normal modes in two degree of freedom systems. Int. $J$. Non-Linear Mech. 8, 161 (1973).

11. G. Pecelli and E. S. Thomas, Normal modes, incoupling, and stability for a class of nonlinear oscillators. Quart. of Appl. Math. 37, 281 (1979).

12. G. Pecelli and E. S. Thomas, Stability of modes for a class of non-linear planar oscillators. Int. J. Non-Linear Mech. 15, 57 (1980).

13. A. F. Vakakis and R. Rand, Normal modes and global dynamics of a two-degree-of-freedom non-linear system-I. Low energies; --II. High energies. Int. J. Non-Linear Mech. 27, 861, 875 (1992).

14. E. L. Ince, Ordinary Differential Equations. Longmans Green, London (1926).

15. V. A. Yakubovich and V. M. Starzhinskii, Linear Differential Equations with Periodic Coefficients, Vols. I and II. J. Wiley, New York (1975).

16. W. Magnus and S. Winkler, Hill's Equation. Interscience Publishers, New York (1966).

17. H. Bateman and A. Erdelyi, Higher Transendental Functions, Vol. 3. Mc Graw-Hill, New York (1955).

18. R. Bellman, Introduction to Matrix Analysis. Mc Graw-Hill, New York (1960).

19. C. S. Hsu, On a restricted class of coupled Hill's equations and some application. Trans. ASME Ser. E. J. Appl. Mech. 28, 551 (1961).

20. H. Bateman and A. Erdelyi, Higher Transendental Functions, Vol. 1. Mc Graw-Hill, New York (1952).

21. A. L. Zhupiev and Yu. V. Mikhlin, Stability and branching of the normal modes of vibrations of the nonlinear systems. PMM USSR 45, 450 (1981).

22. R. K. Bullough and P. J. Caudrey (Eds), Solitons. Springer-Verlag, Berlin (1980).

23. V. E. Zakharov et al., Solitons Theory. Inverse Scattering Method (edited by S. P. Novikov). Nauka, Moscow (1980). [In Russian.]

24. A. L. Zhupiev and Yu. V. Mikhlin, Conditions for finiteness of the number of instability zones in the problem of normal vibrations of non-linear systems. PMM USSR 48, 486 (1984).

25. E. T. Whittaker, Analytical Dynamics of Particles and Rigid Bodies, 4th edition. Cambridge University Press, Cambridge (1937).

26. J. K. Moser, Lectures on Hamiltonian Systems. Courant Inst. of Math. Science, New York (1968).

27. C. L. Zigel and J. K. Moser, Lectures on Celestial Mechanics. Springer-Verlag, Berlin (1971). 